https://doi.org/10.15407/ujpe65.10.939

V.YU. MALYSHEV, I.V. ZAVISLYAK, G.A. MELKOV, M.O. POPOV, O.V. PROKOPENKO

Taras Shevchenko National University of Kyiv,

Faculty of Radiophysics, Electronics, and Computer Systems

(64/13, Volodymyrs'ka Str., Kyiv 01601, Ukraine)

\title{
MICROWAVE MAGNON-PLASMON-POLARITONS IN THE FERROMAGNETIC METAL-SCREENED INSULATOR STRUCTURE
}

\begin{abstract}
A possibility for surface magnon-plasmon-polaritons (SMPPs)-coupled microwave oscillations of magnetization, electron density, and electromagnetic field-to exist in real ferromagnetic metal-insulator-ideal non-magnetic metal structures has been analyzed theoretically. The developed theory predicts that the effective formation of SMPPs is possible only at certain values of the external dc magnetic field and must be accompanied by a shift in the characteristic frequency of the resonance plasmon-polariton systems. A theoretical estimation of the frequency shift for SMPPs in the structure "surface electromagnetic wave resonator made of permalloyvacuum-ideal metal" gives a value of $\pm 45 \mathrm{MHz}$ for a resonator with a characteristic frequency of $10 \mathrm{GHz}$, which seems sufficient for this effect to be observed experimentally.

Ke ywords: magnon, plasmon, polariton, ferromagnetic metal, surface electromagnetic wave resonator, microwave frequency range.
\end{abstract}

\section{Preface}

Intensive studies of magnetic and spin-dependent phenomena in solids started about 60-70 years ago. Till now, they continue to be one of the most challenging and promising areas of research in modern physics. A substantial contribution to the development of this scientific domain has been made by Corresponding Member of the NAS of Ukraine, Dr. Sci. in physics and mathematics, Professor Serhiy Mykhailovych Ryabchenko. The scientific works of Serhiy Mykhailovych, which have become classical long ago (see, e.g., work [1]), were devoted to the study of spin-dependent effects and magnetic resonances in solids, magnetic properties of superconductors, magnetic materials, and nanostructures, magneto-optical effects, and so forth.

Taking an opportunity, we sincerely congratulate Serhiy Mykhailovych on his 80th birthday. We express our gratitude for his active support and cooperation for many years. We wish him good health, even larger creative success, and new talented disciples.

(C) V.YU. MALYSHEV, I.V. ZAVISLYAK, G.A. MELKOV, M.O. POPOV, O.V. PROKOPENKO, 2020

ISSN 2071-0194. Ukr. J. Phys. 2020. Vol. 65, No. 10
Being a scientist with a wide scope of scientific interests, Serhiy Mykhailovych has always been interested in the latest trends in science and tried to implement them into practice. Proceeding from the same principle, we hope that this work will be of interest to many readers. It lies at the interfaces between three physical domains, namely, radio physics, magnetism, and plasmonics, and corresponds to the modern interdisciplinary approach when considering the electrodynamic properties of metal-insulator layered structures.

\section{Introduction}

The existence of surface electromagnetic waves (SEMWs) was theoretically predicted by A. Sommerfeld [2] and J. Zenneck [3] at the end of the 19th century and the beginning of the 20th one. Since then, for more than 100 years, the properties of the SEMWs and the related quasiparticles - surface polaritons - have been studied both theoretically and experimentally in various intervals of the electromagnetic spectrum, ranging from radio to optical frequencies $[4,5]$. Later, those studies brought about the creation of the surface electromagnetic wave resonator (SEMWR) operating in the microwave fre- 


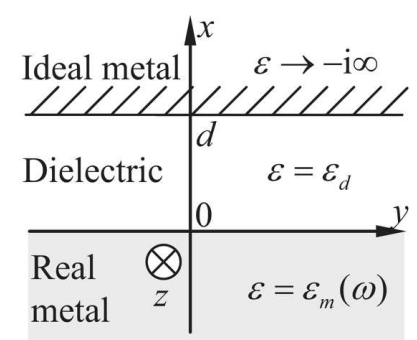

Fig. 1. Three-layer structure real metal-non-magnetic insulator-ideal metal. $\varepsilon$ is the relative dielectric permittivity of the medium: $\varepsilon=\varepsilon_{m}(\omega)$ for the real metal, $\varepsilon=\varepsilon_{d}$ for the insulator, and $\varepsilon \rightarrow-\infty \sqrt{-1}$ for the ideal metal

quency interval $[6,7]$. This system turned out convenient for developing various superconducting devices with Josephson junctions [8-11] (see also recent reviews $[12,13])$.

The theoretical prediction of surface plasmons [14], the quasiparticles that describe collective oscillations of the electron density near the conducting-medium boundary, became a powerful impetus for intensifying the SEMW studies. It was found that the electromagnetic field of the SEMW, when acting on the conductor surface, can generate surface plasmons at the latter, which can excite, in turn, surface polaritons [4, 5, 15-17]. To describe those processes, new quasiparticles, surface plasmon-polaritons (SPPs), were introduced [5, 15-17].

The study of various SPP systems during the last 60 years has led to the appearance of a new branch of science and engineering such as plasmonics [5, 1521]. Today, the study of the properties of SPPs is one of the main tasks of modern physics [18-21]. It is so because, in particular, SPPs are highly promising from the viewpoint of their application in communication systems, medicine, and sensor devices, for measuring the electrophysical parameters of materials, developing the nanotechnology, and so on [5, 15-21].

The properties of SPPs at the interface between a non-magnetic conductor and a non-magnetic insulator were discussed in plenty of papers, textbooks, and monographs $[4,5,16-20]$. At the same time, analogous studies of SPPs in magnetic media are either quite fragmentary or at the very beginning stage, although such systems are very interesting owing to the possibility to considerably change the properties of the SEMWs and SPPs at the interface between the media by means of magnetic fields. Among the interesting works in this area of plasmonics, it is ex- pedient to mention the study of SPPs in the structures with an artificial "left-handed" material [22,23], graphene/antiferromagnet heterostructures [24], on the surface of $\mathrm{MgF}_{2}$ and $\mathrm{FeF}_{2}$ antiferromagnets [25], in multilayer magnetic structures [21, 26, 27], layered metamaterials [28], and so forth. It is also necessary to mention work [29], where the spectrum of quasiparticles in a thin ferrite layer located on the metal surface was researched.

As far as we know, no detailed study of the properties of SPPs in the structure real ferromagnetic metal-non-magnetic insulator-ideal metal (RFMNMI-IM, Fig. 1) has been carried out till now. In particular, the existence of magnons inside a real metal in such three-layer structures has not been taken into account. Therefore, the aim of this work was to develop the theory of SPPs in the three-layer RFM-NMI-IM structure taking the presence of magnons in the ferromagnet into account. As will be shown below, such structures allow the appearance of coupled oscillations of the magnetization and the electron density in the ferromagnetic metal and the electromagnetic field of SEMWs at the metal boundary. Such coupled oscillations can be associated with the appearance of new quasiparticles, surface magnon-plasmonpolaritons (SMPPs).

In this work, the theoretical analysis of the properties of microwave SPPs in the structure RFM-NMI$\mathrm{IM}$ (metal screen) is performed in two stages. A simplified problem concerning SPPs in an RFM-NMI-IM waveguide structure that is infinite in the $y z$-plane is solved in Section 3. In Section 4, the shift of the characteristic frequency of SPPs due to their interaction with magnons is calculated using the perturbation theory in the case of the RFM-NMI-IM waveguide structure with finite dimensions in the $y z$-plane (SPP resonator). The results obtained are analyzed by the example of an SEMWR made of permalloy and possessing the resonance oscillation frequency of the fundamental mode equal to $10 \mathrm{GHz}$.

\section{Surface Plasmon-Polaritons in the Real Metal-Screened Insulator Waveguide Structure}

In Fig. 1, the cross-section of the analyzed real metalscreened insulator (RM-SI) waveguide structure is exhibited. The real metal with the complex relative dielectric permittivity $\varepsilon=\varepsilon_{m}(\omega)=\varepsilon_{m}^{\prime}(\omega)-\mathrm{i} \varepsilon_{m}^{\prime \prime}(\omega)$ occupies the half-space $x \leq 0$, and the screen, which 
is made of an ideal metal with the relative dielectric permittivity $\varepsilon \rightarrow-\mathrm{i} \infty$, the half-space $x \geq d ; \omega$ is the cyclic frequency of the electromagnetic field, and $\mathrm{i}=\sqrt{-1}$ is the imaginary unit. Between the real metal and the ideally conducting metal screen, there is a dielectric layer with the complex relative dielectric permittivity $\varepsilon=\varepsilon_{d}=\varepsilon_{d}^{\prime}-\mathrm{i} \varepsilon_{d}^{\prime \prime}$ and the thickness $d$. The metal layers and the dielectric layer are assumed to be infinite in the $y z$-plane. The relative magnetic permeabilities of all media are equal to unity.

Let us assume that SPPs move in the $+z$-direction of the RM-SI waveguide structure. They are characterized by the longitudinal wave number $\beta$ and have a uniform distribution of the electromagnetic field along the $y$-axis. Let the dependence of the SPP electric, $\mathbf{E}$, and magnetic, $\mathbf{H}$, fields on the time $t$ and the longitudinal coordinate $z$ be chosen in the form $(\mathbf{E}, \mathbf{H}) \sim \mathrm{e}^{\mathrm{i}(\omega t-\beta z)}$, where $\omega=2 \pi f$ is the cyclic frequency of the electromagnetic field.

In order to determine the fields $\mathbf{E}$ and $\mathbf{H}$, let us introduce the electric Hertz vector $\boldsymbol{\Phi}=\left\{0,0, \Phi_{z}\right\}$ with the only non-zero component $\Phi_{z}=\Phi(x) \mathrm{e}^{\mathrm{i}(\omega t-\beta z)}$ and apply the known relations $\mathbf{E}=\operatorname{rot} \operatorname{rot} \boldsymbol{\Phi}$ and $\mathbf{H}=$ $=\mathrm{i} \omega \varepsilon \varepsilon_{0}$ rot $\boldsymbol{\Phi}[30]$, which take into account that the vector $\boldsymbol{\Phi}$ is a solution of the equation $\Delta \boldsymbol{\Phi}+k_{0}^{2} \varepsilon \boldsymbol{\Phi}=0$, where $k_{0}=\omega / c, c$ is the speed of light in vacuum, and $\varepsilon_{0}$ is the electric constant.

It is convenient to express the membrane function $\Phi(x)$ in the form

$\Phi(x)= \begin{cases}A \mathrm{e}^{\alpha x}, & x \leq 0, \\ B \sinh [\tau(d-x)], & 0 \leq x \leq d,\end{cases}$

where $\alpha$ and $\tau$ are the transverse wave numbers (in the general case, they are complex-valued), and the coefficients $A$ and $B$ are the wave amplitudes in the real metal and the insulator, respectively. In this case, the SPP field in the real metal is damped, which is typical of SPPs $[4,5,16-18]$, and the tangential components of the field $\mathbf{E}$ automatically turn to zero at the surface of the ideal metal screen (at $x=d$ ).

Taking formula (1) into account, we can obtain expressions for all nonzero components of the electromagnetic field:

- in the real metal $(x \leq 0)$,

$E_{x}=-\mathrm{i} \alpha \beta A \mathrm{e}^{\alpha x} \mathrm{e}^{\mathrm{i}(\omega t-\beta z)}$,

$E_{z}=-\alpha^{2} A \mathrm{e}^{\alpha x} \mathrm{e}^{\mathrm{i}(\omega t-\beta z)}$,

$H_{y}=-\mathrm{i} \omega \varepsilon_{m}(\omega) \varepsilon_{0} \alpha A \mathrm{e}^{\alpha x} \mathrm{e}^{\mathrm{i}(\omega t-\beta z)}$,
- in the insulator $(0 \leq x \leq d)$,

$E_{x}=\mathrm{i} \beta \tau B \cosh [\tau(d-x)] \mathrm{e}^{\mathrm{i}(\omega t-\beta z)}$,

$E_{z}=-\tau^{2} B \sinh [\tau(d-x)] \mathrm{e}^{\mathrm{i}(\omega t-\beta z)}$,

$H_{y}=\mathrm{i} \omega \varepsilon_{d} \varepsilon_{0} \tau B \cosh [\tau(d-x)] \mathrm{e}^{\mathrm{i}(\omega t-\beta z)}$.

From the continuity conditions for the tangential field components $E_{z}$ and $H_{y}$ at the real metal-insulator interface (at $x=0$ ) and the relation $\beta^{2}=k_{0}^{2} \varepsilon_{m}(\omega)+$ $+\alpha^{2}=k_{0}^{2} \varepsilon_{d}+\tau^{2}$, we obtain the following system of equations for the complex wave numbers $\alpha=\alpha^{\prime}+\mathrm{i} \alpha^{\prime \prime}$ and $\tau=\tau^{\prime}+\mathrm{i} \tau^{\prime \prime}$ :

$\left\{\begin{array}{l}\tau \tanh (\tau d)=-\alpha \frac{\varepsilon_{d}}{\varepsilon_{m}(\omega)}, \\ \alpha^{2}-\tau^{2}=k_{0}^{2}\left(\varepsilon_{d}-\varepsilon_{m}(\omega)\right)\end{array}\right.$

From the boundary conditions at $x=0$, it also follows that the wave amplitudes $A$ and $B$ are mutually coupled by the relation

$B=A\left(\frac{\varepsilon_{m}(\omega)}{\varepsilon_{d}}\right)^{2} \frac{\sinh (\tau d)}{\cosh ^{2}(\tau d)}$.

In the general case, only the numerical solution of the system of equations (3) can be obtained. However, if the structure is non-screened $(d \rightarrow \infty)$, the sought solution consists of the known relations for SPPs at the metal-insulator interface [4, 5, 16-18],

$\alpha=k_{0} \sqrt{\frac{-\varepsilon_{m}^{2}(\omega)}{\varepsilon_{m}(\omega)+\varepsilon_{d}}}, \quad \tau=k_{0} \sqrt{\frac{-\varepsilon_{d}^{2}}{\varepsilon_{m}(\omega)+\varepsilon_{d}}}$.

In the case of a structure with screening $(d<\infty)$, for SPPs to exist, it is necessary that the real and imaginary parts of the complex dielectric permittivity of the metal should provide the spatial localization of the SPP electromagnetic field at the real-metal surface. To illustrate this statement, let us consider the dependences of the ratio $\alpha^{\prime} / \alpha^{\prime \prime}$ on $\varepsilon_{m}^{\prime}$ for various values of $\varepsilon_{m}^{\prime \prime}$ (see Fig. 2). The curves depicted in this figure are numerical solutions of the system of equations (3) in the case of the parameter values $\varepsilon_{d}=1$ (air), $d=5 \mathrm{~mm}$, and $\omega /(2 \pi)=10 \mathrm{GHz}$. The ratio $\alpha^{\prime} / \alpha^{\prime \prime}$ is equal, neglecting a constant factor, to the quantity $\Lambda / \delta$, i.e. the ratio between the spatial period of field oscillations in the metal, $\Lambda$, and the characteristic depth of the electromagnetic field penetration into the metal (the skin-layer thickness), $\delta$. Therefore, it characterizes rather well the behavior of SPPs near the 


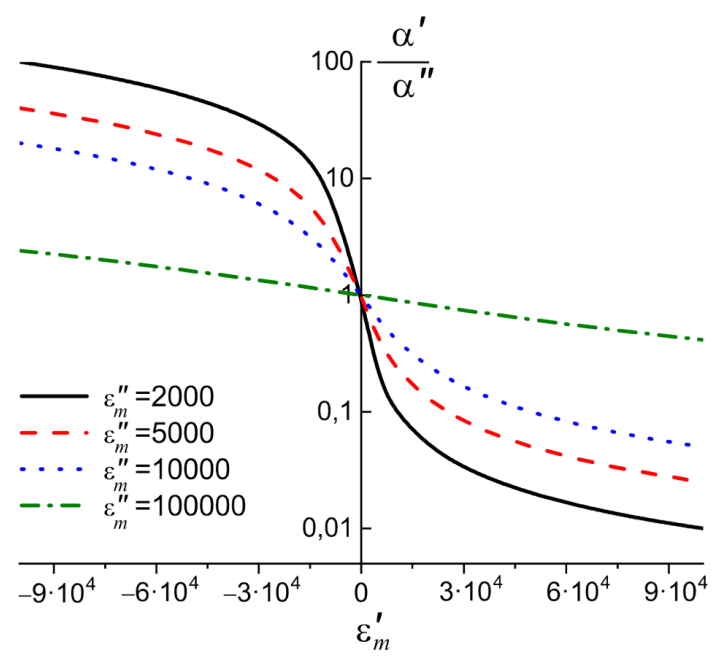

Fig. 2. Dependences of the ratio $\alpha^{\prime} / \alpha^{\prime \prime}$ on $\varepsilon_{m}^{\prime}\{\mathrm{m}\}$ calculated for various $\varepsilon_{m}^{\prime \prime}$-values on the basis of a numerical solution of the system of equations (3). The calculation parameters are $\varepsilon_{d}=1, d=5 \mathrm{~mm}$, and $\omega /(2 \pi)=10 \mathrm{GHz}$

metal-insulator interface. At $\Lambda / \delta \gg 1$ (i.e. $\delta \ll \Lambda$ ), the SPP electromagnetic field is strongly localized near the real-metal surface, whereas, at $\Lambda / \delta \ll 1$ (i.e. $\delta \gg \Lambda$ ), the electromagnetic field penetrates deeply into the real metal, and its amplitude decays slowly. As one can see from Fig. 2 , if $\varepsilon_{m}^{\prime}<0$, the ratio $\Lambda / \delta>1$ at any $\varepsilon_{m}^{\prime \prime}$, i.e. the electromagnetic wave at the real metal-insulator interface is a true surface wave. In this case, if the absolute value of $\varepsilon_{m}^{\prime}$ increases or $\varepsilon_{m}^{\prime \prime}$ decreases, the degree of field localization grows.

Note also the following important circumstance: in the case where $\varepsilon_{m}^{\prime \prime} \gg\left|\varepsilon_{m}^{\prime}\right|$, the ratio $\Lambda / \delta \rightarrow 1$ irrespective of the $\varepsilon_{m}^{\prime}$ sign. Hence, the condition $\varepsilon_{m}^{\prime}<0$, which is typical of the SPP optical interval $[4,5,16-$ 18 , is, strictly speaking, only the sufficient condition of localization of the SPP electromagnetic field at a real-metal surface. In the opposite case $\varepsilon_{m}^{\prime}>0$, the wave can also be the surface one, if the additional condition $\varepsilon_{m}^{\prime \prime} \gg\left|\varepsilon_{m}^{\prime}\right|$ is satisfied. At optical frequencies, the inequality $\varepsilon_{m}^{\prime \prime} \gg\left|\varepsilon_{m}^{\prime}\right|$ is not obeyed, and the inequality $\varepsilon_{m}^{\prime}<0$ is simultaneously the necessary and sufficient condition for SPPs to exist in this case. However, in the microwave frequency interval, which is considered in this work, it is the relation $\varepsilon_{m}^{\prime \prime} \gg\left|\varepsilon_{m}^{\prime}\right|$ that is true for real metals described by the Drude model.

Note also that Eqs. (3) and (5) give rise to the following important fact. In the unscreened structure $(d \rightarrow \infty)$, the electromagnetic field of a wave outside the real metal (at $x \geq 0$ ) changes rather weakly, and the penetration depth of the microwave field into the insulator can reach a value of $1 / \tau^{\prime} \sim 10 \div 100 \mathrm{~m}$. However, the presence of an ideal metal screen substantially enhances the localization of the SPP electromagnetic field at the real-metal surface $\left(1 / \tau^{\prime} \sim d\right)$. Hence, when applying microwave systems with SPPs in practice, it is appropriate to screen them. In so doing, by changing the distance $d$ between the real metal and the screen, it is possible to considerably affect the localization of the SPP electromagnetic field at the real-metal surface.

In the framework of the Drude model, the relative dielectric permittivity of a real metal, $\varepsilon_{m}(\omega)$, can be written as follows $[5,16,17,19,31]$ :

$\varepsilon_{m}(\omega)=1-\frac{\omega_{p}^{2}}{\omega^{2}-\mathrm{i} \omega \Gamma}=\varepsilon_{m}^{\prime}(\omega)-\mathrm{i} \varepsilon_{m}^{\prime \prime}(\omega)$,

$\varepsilon_{m}^{\prime}(\omega)=1-\frac{\omega_{p}^{2}}{\omega^{2}+\Gamma^{2}}$,

$\varepsilon_{m}^{\prime \prime}(\omega)=\frac{\omega_{p}^{2} \Gamma}{\omega\left(\omega^{2}+\Gamma^{2}\right)}$,

where

$\omega_{p}=\sqrt{\frac{n e^{2}}{\varepsilon_{0} m_{e}}}$

is the cyclic plasma frequency of the electron gas in a conducting medium with the electron concentration $n$ and the specific conductance $\sigma$,

$\Gamma=\frac{n e^{2}}{\sigma m_{e}}$

is the frequency of electron collisions in plasma, $e$ is the elementary electric charge, and $m_{e}$ is the electron mass. An important issue arises concerning the correctness of the SPPs description at the boundary of a real metal with the relative dielectric permittivity $\varepsilon_{m}(\omega)$ making use of Eqs. (6) in the microwave frequency interval (as a rule, formulas (6) are applied when describing the properties of metals in the terahertz, infrared, and optical ranges $[4,5,16,17,19,31])$. The Drude model (6) is an approximation and describes the behavior of metals well, only if the frequencies are sufficiently high $\left(\gtrsim 10^{-2} \Gamma\right)[5,16,17,19]$. At the same time, it follows from Eqs. (6) that $\varepsilon_{m}^{\prime \prime}(\omega) \rightarrow \infty$ as $\omega \rightarrow 0$, which demonstrates the model incorrectness. There are more adequate models, which correctly describe

ISSN 2071-0194. Ukr. J. Phys. 2020. Vol. 65, No. 10 
the behavior of $\varepsilon_{m}(\omega)$, e.g., the Lindhard quantummechanical model [32]. Nevertheless, below, we will use only the Drude model. At the same time, we will evaluate the minimum frequency of the electromagnetic field, $\omega_{\min }$, for which the application of formulas (6) still remains valid.

It is evident that the condition $\varepsilon_{m}^{\prime}(\omega)=0$ corresponds to the case where the electromagnetic field frequency $\omega$ coincides with the eigenfrequency of one of the resonances that exist in the real metal, because the presence of such a resonance appreciably modifies the response of the electron gas to an external electromagnetic influence [31]. In particular, one of such resonances can be the resonance associated with the excitation of longitudinal acoustic phonons with the characteristic frequency $\omega_{a}=2 \pi v_{a} / \lambda_{a}$, the wavelength $\lambda_{a}$, and the sound velocity $v_{a}$ [31]. For ordinary metals, $\lambda_{a} \sim 2 \delta$, where $\delta \sim 1 \mu \mathrm{m}$ is the skin layer thickness, and $v_{a} \sim 10^{3} \mathrm{~m} / \mathrm{s}$, which gives $\omega_{a}=\omega_{\min } \sim 10^{10} \mathrm{~s}^{-1}$. Therefore, the application of formulas (6) to describe SPPs in the framework of the Drude theory is correct in the frequency interval $\omega_{\min }<\omega<\omega_{\max }$, where $\omega_{\min } \sim 10^{10} \mathrm{~s}^{-1}$ and $\omega_{\max }=\sqrt{\omega_{p}^{2}-\Gamma^{2}} \approx \omega_{p} \sim 10^{16} \mathrm{~s}^{-1}(\Gamma \sim$ $\sim 10^{14} \mathrm{~s}^{-1}$ and $\left.\omega_{p} \sim 10^{16} \mathrm{~s}^{-1}\right)$. Thus, the conditions $\varepsilon_{m}^{\prime \prime}(\omega)>\left|\varepsilon_{m}^{\prime}(\omega)\right|$ can be easily satisfied within almost the whole microwave frequency interval for which $\omega_{\min }<\omega \ll \Gamma \ll \omega_{\max }<\omega_{p}$.

\section{Surface Magnon-Plasmon-Polaritons in the Real Ferromagnetic Metal-Screened Insulator Resonance Structure}

Let the waveguide structure, the cross section of which is shown in Fig. 1, possess the finite size $w$ along the $z$-axis. Hence, it is an open waveguide resonator [30]. In this structure, owing to the interference between the waves incident on the structure ends (at $z=0$ and $w$ ) and the waves reflected from them, microwave oscillations may arise. The electromagnetic field of those oscillations will be mainly localized in the region $0<z<w$ and will be characterized by the real longitudinal wave number $\beta \approx n \pi / w$ $(n \in \mathbb{Z})$. When writing the parameter $\beta$ in this form, we actually neglect the leakage of the SPP electromagnetic field beyond the region $0 \leq z \leq w$ and the losses occurring at the wave propagation in the directions $\pm z$. Let us additionally assume that the structure size along the $y$-axis is also finite and equal to $l$ (i.e. the electromagnetic field is localized in the region $0 \leq y \leq l)$. Thus, unlike the problem considered in Section 3, now the dimensions of all elements in the RFM-NMI-IM structure in the $y z$-plane are finite $(l \times w)$. Therefore, the dependence of the SPP electromagnetic field on the coordinates $y$ and $z$ can be formally characterized by the wave numbers $m \pi / l$ and $n \pi / w$, respectively, where the integers $(m, n) \in \mathbb{Z}$ define the oscillation mode in the resonance structure under consideration.

Provided the conditions $d \rightarrow \infty$ and $\varepsilon_{d}=1$, the examined resonance structure is the plate of a real metal with finite lateral dimensions $l \times w$ and is located in the free space. This structure is the simplest case of the SEMWR on the basis of a conducting plate (film) with a finite thickness $[6,8,9,12,13]$. But if $d<\infty$ and $\varepsilon_{d} \geq 1$, the RFM-NMI-IM resonance structure can be identified with an SEMWR (layer of a real metal on a dielectric substrate) arranged on the wall surface in a rectangular waveguide (the ideal metal screen). Let us consider the metal screen to be an ideal conductor, if its conductivity is higher than the conductivity of a real metal by at least an order of magnitude. Note that just such SEMWRs on the basis of a conducting film deposited onto a dielectric substrate are used in practice. For the efficient excitation of oscillations in the SEMWR, the latter is mounted inside a rectangular waveguide [6-13].

A complete theoretical analysis of the microwave properties of the SEMWR is rather complicated [6$8,12,13]$. Therefore, let us only consider the simplified model introduced in Section 3. This model can approximately describe the behavior of the fundamental SEMWR mode, the mode $\mathrm{E}_{01}$, for which $m=0$ and $n=1$. For this purpose, let us make the following substitutions in expressions (2): the substitution $\mathbf{e}^{-\mathrm{i} \beta z} \rightarrow \sin (\beta z)$ in the components $E_{z}$ and $H_{y}$ and the substitution $\mathbf{e}^{-\mathrm{i} \beta z} \rightarrow \cos (\beta z)$ in the component $E_{x}$, assuming $\beta \approx \pi / w$. In addition, we should take into account that the distribution of the electromagnetic field in the RM-SI resonance structure does not depend on the coordinate $y$ (as is indicated by the index $m=0$ for the fundamental SEMWR mode $\mathrm{E}_{01}$; note that this mode was experimentally determined to be quasi-uniform in the SEMWRs $[6,8,9,13])$. Taking the aforesaid into account, we obtain the following expressions for the electromagnetic field components at the fundamental mode of oscillations in the SEMWR, $\mathrm{E}_{01}$ : 
- in the real metal $(x \leq 0)$,

$$
\begin{aligned}
& E_{x}=-\mathrm{i} \alpha \beta A \mathrm{e}^{\alpha x} \cos \left(\frac{\pi}{w} z\right) \mathrm{e}^{\mathrm{i} \omega t}, \\
& E_{z}=-\alpha^{2} A \mathrm{e}^{\alpha x} \sin \left(\frac{\pi}{w} z\right) \mathrm{e}^{\mathrm{i} \omega t} \\
& H_{y}=-\mathrm{i} \omega \varepsilon_{m}(\omega) \varepsilon_{0} \alpha A \mathrm{e}^{\alpha x} \sin \left(\frac{\pi}{w} z\right) \mathrm{e}^{\mathrm{i} \omega t},
\end{aligned}
$$

- in the insulator $(0 \leq x \leq d)$,

$$
\begin{aligned}
& E_{x}=\mathrm{i} \beta \tau B \cosh [\tau(d-x)] \cos \left(\frac{\pi}{w} z\right) \mathrm{e}^{\mathrm{i} \omega t}, \\
& E_{z}=-\tau^{2} B \sinh [\tau(d-x)] \sin \left(\frac{\pi}{w} z\right) \mathrm{e}^{\mathrm{i} \omega t}, \\
& H_{y}=\mathrm{i} \omega \varepsilon_{d} \varepsilon_{0} \tau B \cosh [\tau(d-x)] \sin \left(\frac{\pi}{w} z\right) \mathrm{e}^{\mathrm{i} \omega t} .
\end{aligned}
$$

Finally, we assume that the resonance frequency of the fundamental oscillation mode in the examined resonance structure, which enters expressions (7), is known: $\omega=\omega_{0}=2 \pi f_{0}$.

Earlier, the magnetic properties of a real metal were not taken into consideration. Now, let us suppose that there are magnons in the real ferromagnetic metal and they can interact with SPPs that exist at the metal boundary. It is no doubt that such interaction has to modify the electromagnetic field distribution in the resonance RM-SI structure and induce the deviation (shift) of the resonance frequency of the structure, $\omega_{r}$, from $\omega_{0}$.

Assuming that the interaction of magnons with SPPs is rather weak, let us apply the methods of perturbation theory [33] in order to determine a relative shift of the resonance frequency of the structure, $\Delta \omega / \omega_{0}$, where $\Delta \omega=\omega_{r}-\omega_{0}$, and it is supposed that $|\Delta \omega| \ll\left(\omega_{r}, \omega_{0}\right)$. The role of an unperturbed system, in this case, is played by the fundamental SEMWR mode with the frequency $\omega_{0}$. The electromagnetic field of this mode in a resonator undergoes a perturbation, because the relative magnetic permeability of a real metal differs from unity and becomes a tensor quantity $\hat{\mu}$. To describe this perturbation, let us use the known relations [30,33]:

$$
\begin{aligned}
& \frac{\Delta \omega}{\omega_{0}}=\frac{\omega_{r}-\omega_{0}}{\omega_{0}}=-\frac{\Delta W}{W_{0}}, \\
& \Delta W=\mu_{0} \int_{V_{m}} \mathbf{H}_{0}^{*} \Delta \hat{\mu} \mathbf{H} d V=\mu_{0} \int_{V_{m}} \mathbf{H}_{0}^{*} \hat{\chi} \mathbf{H}_{0} d V, \\
& W_{0}=2 \mu_{0} \int_{V_{0}}\left|\mathbf{H}_{0}\right|^{2} d V
\end{aligned}
$$

where $V_{m} \in\{-\infty<x \leq 0,0 \leq y \leq l, 0 \leq z \leq w\}$ is the volume of the metal, where the electromagnetic field and magnons coexist, $V_{0} \in\{-\infty<x \leq d$, $0 \leq y \leq l, 0 \leq z \leq w\}$ is the volume of the system, where only the electromagnetic field is present, $\mathbf{H}_{0}$ is the magnetic field of the unperturbed oscillation mode, $\mathbf{H}$ is the magnetic field of the perturbed oscillation mode, $\Delta \hat{\mu}$ and $\hat{\chi}$ are tensors characterizing the resonator field perturbation, $\Delta W$ is a quantity proportional to the energy change of the electromagnetic field in the volume $V_{m}, W_{0}$ is a quantity proportional to the total energy of the electromagnetic field in the unperturbed resonator, and $\mu_{0}$ is the magnetic constant. Note that, formally, the quantities $\omega_{r}$ and $\omega_{0}$ in Eqs. (8) and in the expressions below should be considered as complex-valued: $\omega_{r}=\omega_{r}^{\prime}+\mathrm{i} \omega_{r}^{\prime \prime}$ and $\omega_{0}=\omega_{0}^{\prime}+\mathrm{i} \omega_{0}^{\prime \prime}$.

Substituting the expressions for the electromagnetic field component $H_{y}$ from Eqs. (7) into Eq. (8c), integrating the result over the volume $V_{0}$, and making use of relations (3) between the wave numbers $\alpha$ and $\tau$, we obtain

$$
\begin{aligned}
& W_{0}=|A|^{2} k_{0}^{2} l w \varepsilon_{0}\left|\varepsilon_{m}(\omega)\right|^{2}|\alpha|^{2} \times \\
& \times\left[\frac{1}{2 \alpha^{\prime}}+\frac{\tau \tanh (\tau d)-\tau^{*} \tanh \left(\tau^{*} d\right)}{\tau^{2}-\tau^{* 2}}\right]= \\
& =|A|^{2} k_{0}^{2} l w \varepsilon_{0}\left|\varepsilon_{m}(\omega)\right|^{2}|\alpha|^{2}\left[\frac{1}{2 \alpha^{\prime}}+\frac{1}{\left|1+\mathrm{e}^{-2 \tau d}\right|^{2}} \times\right. \\
& \left.\times\left(\frac{1-\mathrm{e}^{-4 \tau^{\prime} d}}{2 \tau^{\prime}}+\frac{\sin \left(2 \tau^{\prime \prime} d\right) \mathrm{e}^{-2 \tau^{\prime} d}}{\tau^{\prime \prime}}\right)\right] .
\end{aligned}
$$

If the distance $d$ between the real metal and the ideal metal screen is short $(\tau d \ll 1)$, this expression can be simplified:

$W_{0}=|A|^{2} k_{0}^{2} l w \varepsilon_{0}\left|\varepsilon_{m}(\omega)\right|^{2}|\alpha|^{2}\left(\frac{1}{2 \alpha^{\prime}}+d\right)$.

In the opposite limit $d \rightarrow \infty$, i.e. for a non-screened system, the expression for $W_{0}$ reads

$$
W_{0}=|A|^{2} k_{0}^{2} l w \varepsilon_{0}\left|\varepsilon_{m}(\omega)\right|^{2}|\alpha|^{2}\left(\frac{1}{2 \alpha^{\prime}}+\frac{1}{2 \tau^{\prime}}\right) .
$$

In order to find the quantity $\Delta W$, it is necessary to specify an explicit expression for the magnetic susceptibility tensor $\hat{\chi}$, which, in turn, depends on the spatial orientation of the external dc magnetic field

ISSN 2071-0194. Ukr. J. Phys. 2020. Vol. 65, No. 10 
with the flux density $\mathbf{B}_{\mathrm{dc}}$. If we assume that the external magnetic field lies in the specimen plane, then, in order to effectively excite magnetic oscillations, it must be directed perpendicular to the microwave field, i.e. along the $z$-axis, $\mathbf{B}_{\mathrm{dc}}=\left\{0,0, B_{\mathrm{dc}}\right\}$. In this case, the tensor $\hat{\chi}$ looks like [33]

$$
\begin{aligned}
\hat{\chi} & =\left(\begin{array}{ccc}
\chi_{x x} & -\mathrm{i} \chi_{a} & 0 \\
\mathrm{i} \chi_{a} & \chi_{y y} & 0 \\
0 & 0 & 0
\end{array}\right), \\
\chi_{x x} & =\frac{\omega_{M}\left(\omega_{H}+\omega_{M} N_{y y}\right)}{\left(\omega_{H}+\omega_{M} N_{y y}\right)\left(\omega_{H}+\omega_{M} N_{x x}\right)-\omega^{2}}, \\
\chi_{y y} & =\frac{\omega_{M}\left(\omega_{H}+\omega_{M} N_{x x}\right)}{\left(\omega_{H}+\omega_{M} N_{y y}\right)\left(\omega_{H}+\omega_{M} N_{x x}\right)-\omega^{2}}, \\
\chi_{a} & =\frac{\omega \omega_{M}}{\left(\omega_{H}+\omega_{M} N_{y y}\right)\left(\omega_{H}+\omega_{M} N_{x x}\right)-\omega^{2}}, \\
\omega_{H} & =\gamma B_{\mathrm{dc}}-\omega_{M} N_{z z}, \omega_{M}=\gamma \mu_{0} M_{0},
\end{aligned}
$$

$\gamma \approx 2 \pi \times 28 \mathrm{GHz} / \mathrm{T}$ is the absolute value of the gyromagnetic ratio; $N_{x x}, N_{y y}$, and $N_{z z}$ are the demagnetizing coefficients; and $M_{0}$ is the saturation magnetization of the ferromagnet. Taking Eq. (10a) into account, the integrand $\mathbf{H}_{0}^{*} \hat{\chi} \mathbf{H}_{0}$ in Eq. (8b) is reduced to $\chi_{y y}\left|H_{y}\right|^{2}$, where the field component $H_{y}$ is determined by Eq. $(7 \mathrm{c})$, and $\chi_{y y} \equiv \chi_{y y}\left(B_{\mathrm{dc}}\right)$. Then, calculating the integral in Eq. (8b), we obtain

$\Delta W=\frac{\chi_{y y}}{4 \alpha^{\prime}}|A|^{2} k_{0}^{2} l w \varepsilon_{0}\left|\varepsilon_{m}(\omega)\right|^{2}|\alpha|^{2}$.

As a result, the relative shift of the complex frequency of the resonator due to the existence of magnons in the real metal equals

$$
\begin{aligned}
& \frac{\Delta \omega}{\omega_{0}}=-\frac{\chi_{y y}}{4 \alpha^{\prime}}\left[\frac{1}{2 \alpha^{\prime}}+\frac{1}{\left|1+\mathrm{e}^{-2 \tau d}\right|^{2}} \times\right. \\
& \left.\times\left(\frac{1-\mathrm{e}^{-4 \tau^{\prime} d}}{2 \tau^{\prime}}+\frac{\sin \left(2 \tau^{\prime \prime} d\right) \mathrm{e}^{-2 \tau^{\prime} d}}{\tau^{\prime \prime}}\right)\right]^{-1} .
\end{aligned}
$$

Taking into account that $\alpha^{\prime} d \sim d / \delta \gg 1$ in the microwave frequency interval, this expression can be simplified:

$$
\frac{\Delta \omega}{\omega_{0}} \approx-\frac{\chi_{y y}}{4 \alpha^{\prime} d}
$$

In the case of non-screened structure, Eq. (12) becomes

$$
\frac{\Delta \omega}{\omega_{0}} \approx-\frac{\chi_{y y}}{2\left(1+\frac{\alpha^{\prime}}{\tau^{\prime}}\right)} \approx-\frac{\chi_{y y}}{2} \frac{\left|\varepsilon_{d}\right|}{\left|\varepsilon_{m}(\omega)\right|} .
$$

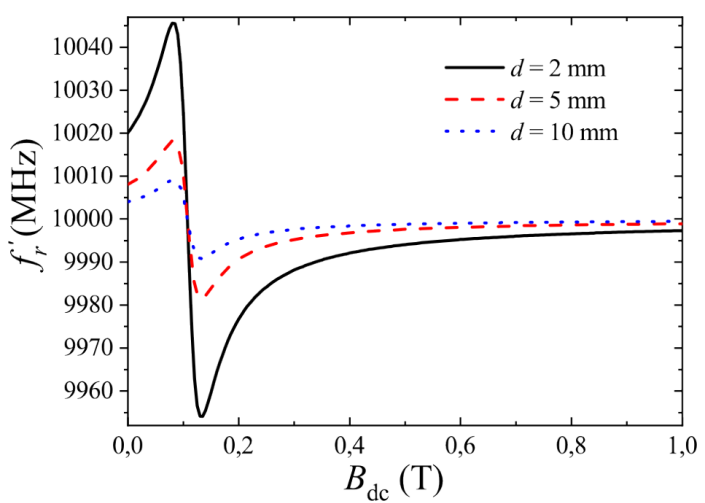

Fig. 3. Dependences of the frequency $f_{r}^{\prime}=\omega_{r}^{\prime} /(2 \pi)$ of the plasmon-polariton resonance at the permalloy-vacuum interface on the flux density of the external dc magnetic field $B_{\mathrm{dc}}$ applied in parallel to the ferromagnet surface calculated for various distances $d$ between permalloy and the ideal metal screen

Now, performing the substitution $B_{\mathrm{dc}} \rightarrow B_{\mathrm{dc}}+$ $+\mathrm{i} \Delta B / 2$ in the expression for $\chi_{y y}$, we obtain the following final formula for the variation of the real resonance frequency of the structure due to the interaction between SPPs and magnons in the real metal:

$$
\begin{aligned}
& \frac{\omega_{r}^{\prime}-\omega_{0}^{\prime}}{\omega_{0}^{\prime}}=-\operatorname{Re}\left\{\frac { \chi _ { y y } ( B _ { \mathrm { dc } } + \mathrm { i } \Delta B / 2 ) } { 4 \alpha ^ { \prime } } \left[\frac{1}{2 \alpha^{\prime}}+\right.\right. \\
& \left.\left.+\frac{1}{\left|1+\mathrm{e}^{-2 \tau d}\right|^{2}}\left(\frac{1-\mathrm{e}^{-4 \tau^{\prime} d}}{2 \tau^{\prime}}+\frac{\sin \left(2 \tau^{\prime \prime} d\right) \mathrm{e}^{-2 \tau^{\prime} d}}{\tau^{\prime \prime}}\right)\right]^{-1}\right\} .
\end{aligned}
$$

The results of calculations by formula (15) for permalloy $\mathrm{Ni}_{81} \mathrm{Fe}_{19}$, as a real metal, are shown in Fig. 3. The calculations were carried out for a specimen in the form of a plate and magnetized along the longer size $w$, so that $N_{z z} \approx 0, N_{y y} \approx 0, N_{x x} \approx 1$, and

$\chi_{y y}=\frac{\omega_{M}\left(\omega_{H}+\omega_{M}\right)}{\omega_{H}\left(\omega_{H}+\omega_{M}\right)-\omega^{2}}$.

The other calculation parameters were as follows: $\mu_{0} M_{0}=1080 \mathrm{mT}, \Delta B=50 \mathrm{mT}, \gamma=2 \pi \times 28 \mathrm{GHz} / \mathrm{T}$, the unperturbed plasmon-polariton resonance frequency $f_{0}^{\prime}=\omega_{0}^{\prime} /(2 \pi)=10 \mathrm{GHz}$, and the distance from the real metal surface to the ideal metal screen $d=2,5$, and $10 \mathrm{~mm}$. The dielectric permittivity of the metal $\varepsilon_{m}(\omega)$ was determined in the framework of the Drude model (6). The required parameters - the plasma frequency and the relaxation frequency - were taken as for nickel [34]. 


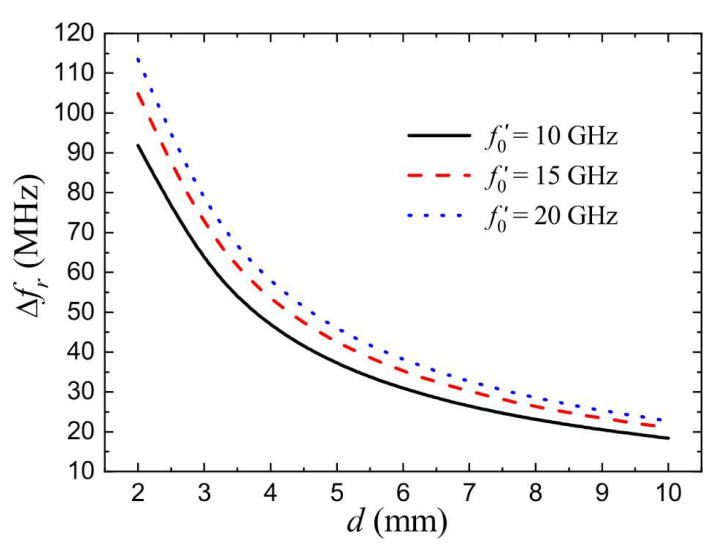

Fig. 4. Dependences of the maximum interval of the magnetically induced tuning of the plasmon-polariton resonance frequency, $\Delta f_{r}=f_{r, \max }^{\prime}-f_{r, \min }^{\prime}$, on the distance $d$ between the ideal metal screen and the ferromagnet-based SEMWR. Calculations were carried out for the fundamental mode of oscillations in SEMWRs with various unperturbed frequencies $f_{0}^{\prime}$

From Fig. 3, one can see that appreciable changes in the plasmon-polariton resonance frequency $f_{r}^{\prime}$ by $\Delta f_{r}=f_{r, \text { max }}^{\prime}-f_{r, \text { min }}^{\prime} \approx 20 \div 90 \mathrm{MHz}$, where $f_{r, \text { max }}^{\prime}$ and $f_{r, \text { min }}^{\prime}$ are the maximum and minimum, respectively, frequencies of the plasmon-polariton resonance, are observed at rather low fields $B_{\mathrm{dc}} \approx$ $\approx 50 \div 250 \mathrm{mT}$. Obviously, in this interval of the magnetization field induction values $B_{\mathrm{dc}}$, if the magnon frequency becomes close to the SPP frequency, there arises an intense interaction between those quasiparticles (the electromagnetic field of SEMWs strongly interacts with the magnetization of a ferromagnetic metal). As a result, in the region where the interaction is the strongest, the corresponding oscillations of the electromagnetic field, electron density, and magnetization partially hybridize with one another. One of the possible ways to explain the appearance of such hybridized oscillations may consist in the formal introduction of a magnon-plasmon-polariton resonance, i.e. new quasiparticles, SMPPs. A detailed analysis of their properties requires a separate study.

Figure 4 demonstrates the dependences of the maximum of the frequency tuning interval of the magnonplasmon-polariton resonance $\Delta f_{r}$ for an SEMWR fabricated from permalloy $\mathrm{Ni}_{81} \mathrm{Fe}_{19}$ on the distance between the SEMWR and the ideal conducting screen calculated for various values of the unperturbed resonator frequency $f_{0}^{\prime}$. The value of $\Delta f_{r}$ was deter- mined as the difference between the values of the frequencies $f_{r, \max }^{\prime}$ and $f_{r, \text { min }}^{\prime}$ corresponding to the extrema in the dependences $f_{r}^{\prime}\left(B_{\mathrm{dc}}\right)$, the latter being similar to those shown in Fig. 3. One can see that, as the screen approaches the ferromagnetic metal, the efficiency of the control over the resonator frequency by means of the magnetic field increases. From the physical viewpoint, this occurs due to the growth of the relative contribution made by the electromagnetic field located in the metal to the total resonator energy. Actually, this effect has already been partially discussed in Section 3, where it was noted that the shorter distances $d$ between the screen and the real metal force the SPP electromagnetic field to be localized at the real-metal surface. As a result, the interaction between magnons and SPPs becomes stronger, which gives rise to a more efficient SMPP formation. Note also that if the unperturbed frequency $f_{0}^{\prime}$ increases, the indicated tuning interval $\Delta f$ grows monotonically, but this dependence is much weaker than the dependence $\Delta f_{r}(d)$.

Finally, it is important to emphasize that the characteristic shift $\Delta f$ of the frequency induced by the appearance of SMPPs is rather large (the magnitude of the effect $\Delta f_{r} / f_{0}^{\prime} \approx 90 \mathrm{MHz} / 10 \mathrm{GHz} \sim 1 \%$ ), so it can be measured experimentally. In the case of success, a similar method of experimental research could be used in the future to measure the dielectric permittivities of metals in the microwave frequency interval.

\section{Conclusions}

In this work, a possibility for microwave surface magnon-plasmon-polaritons (SMPPs) to be formed at the interface between a real ferromagnetic metal with relative dielectric permittivity $\varepsilon_{m}=\varepsilon_{m}^{\prime}-\mathrm{i} \varepsilon_{m}^{\prime \prime}$ and a screened insulator has been shown theoretically. It is found that, unlike the optical frequency interval, where the existence of surface plasmon-polaritons (SPPs) is possible only if $\varepsilon_{m}^{\prime}<0$, SPPs can exist in the microwave interval irrespective of the $\varepsilon_{m}^{\prime}$-sign, but provided that $\varepsilon_{m}^{\prime \prime} \gg\left|\varepsilon_{m}^{\prime}\right|$. It is predicted theoretically that an appreciable change of the plasmonpolariton resonance frequency should be observed in the real ferromagnetic metal-insulator-ideal metal screen resonance structures controlled by an external magnetic field at rather low flux density values 
$B_{\mathrm{dc}} \sim 100 \mathrm{mT}$. This effect is explained by the formation of SMPPs. Its magnitude should become larger at shorter distances between the screen and the realmetal surface and reach a value of about $1 \%$. If this effect is confirmed experimentally, it could be applied to measure the dielectric permittivities of metals in the microwave frequency range.

The work was supported by the Ministry of Education and Science of Ukraine (budget themes No. 18BF052-01M and 19BF052-01), the National Academy of Sciences of Ukraine (grant No. $1 F$ ).

1. A.M. Pogorilyi, S.M. Ryabchenko, O.I. Tovstolytkin. Spintronics. Main phenomena. Development trends. Ukr. Fiz. Zh. Oglyad. 6, 37 (2010) (in Ukrainian).

2. A. Sommerfeld. Über die Fortpflanzung elektrodynamischer Wellen längs eines Drahtes. Ann. Phys. Chem. 303, 233 (1899).

3. J. Zenneck. Über die Fortpflanzung ebener elektromagnetischer Wellen längs einer ebenen Leiterfläche und ihre Beziehung zur drahtlosen Telegraphie. Ann. Phys. 328, 846 (1907).

4. Surface Polaritons: Electromagnetic Waves at Surfaces and Interfaces. Edited by V.M. Agranovich, D.L. Mills (North-Holland, 1982).

5. E.L. Albuquerque, M.G. Cottam. Polaritons in Periodic and Quasiperiodic Structures (Elsevier, 2004).

6. G.A. Melkov, Y.V. Egorov, O.M. Ivanyuta, V.Y. Malyshev, H.K. Zeng, Kh. Wu, J.Y. Juang. HTS surface wave resonators. J. Supercond. 13, 95 (2000).

7. G.A. Melkov, A.V. Prokopenko, V.N. Raksha. Rarefaction of the natural oscillation spectrum of a surface wave resonator. Radioelectr. Commun. Syst. 47, 20 (2004).

8. G.A. Melkov, O.M. Ivanyuta, O.V. Prokopenko, V.M. Raksha, A.M. Klushin, M. Siegel. Embedding of Josephson junctions in the surface wave resonator in the Kaband. In: Proceedings of the 4th International Khar'kov Symposium "Physics and Engineering of Millimeter and Sub-Millimeter Waves" (MSMW'2001, 4-9 June 2001, Khar'kov, Ukraine) (2001), Vol. 1, p. 363.

9. G.A. Melkov, A.M. Klushin, O.D. Poustylnik, O.V. Prokopenko, V.M. Raksha. Irradiation of HTS Josephson junctions with the surface wave resonator. In: Proceedings of the 5th International Khar'kov Symposium "Physics and Engineering of Millimeter and Sub-Millimeter Waves" (MSMW'2004, 21-26 June 2004, Khar'kov, Ukraine) (2004), Vol 2, p. 128.

10. O.M. Ivanyuta, O.V. Prokopenko, V.M. Raksha, A.M. Klushin. Microwave detection using Josephson junction arrays integrated in a resonator. Phys. Status Solidi C 2, 1688 (2005).

ISSN 2071-0194. Ukr. J. Phys. 2020. Vol. 65, No. 10
11. O.M. Ivanyuta, O.V. Prokopenko, Ya.I. Kishenko, V.M. Raksha, A.M. Klushin. The effect of the external magnetic field on the current-voltage characteristic of HTS Josephson junction arrays. J. Low Temp. Phys. 139, 97 (2005).

12. O.V. Prokopenko, D.A. Bozhko, V.S. Tyberkevych, A.V. Chumak, V.I. Vasyuchka, A.A. Serga, O. Dzyapko, R.V. Verba, A.V. Talalaevskij, D.V.Slobodianiuk, Yu.V. Kobljanskyj, V.A. Moiseienko, S.V. Sholom, V.Yu. Malyshev. Recent trends in microwave magnetism and superconductivity. Ukr. J. Phys. 64, 888 (2019).

13. V. Malyshev, G. Melkov, O. Prokopenko. Microwave devices based on superconducting surface electromagnetic wave resonator (Review Article). Fiz. Nizk. Temp. 46, 422 (2020).

14. R.H. Ritchie. Plasma losses by fast electrons in thin films. Phys. Rev. 106, 874 (1957).

15. E. Özbay. Plasmonics: Merging photonics and electronics at nanoscale dimensions. Science 311, 189 (2006).

16. S.A. Maier. Plasmonics: Fundamentals and Applications (Springer, 2007).

17. Plasmonics: From Basics to Advanced Topics. Edited by S. Enoch, N. Bonod (Springer, 2012).

18. R.S. Anwar, H. Ning, L. Mao. Recent advancements in surface plasmon polaritons-plasmonics in subwavelength structures in microwave and terahertz regimes. Digit. Commun. Netw. 4, 244 (2018).

19. Surface Electromagnetics: With Applications in Antenna, Microwave, and Optical Engineering. Edited by F. Yang, Y. Rahmat-Samii (Cambridge Univ. Press, 2019).

20. X. Zhang, Q. Xu, L. Xia, Y. Li, J. Gu, Z. Tian, C. Ouyang, J. Han, W. Zhang. Terahertz surface plasmonic waves: a review. Adv. Photon. 2, 014001 (2020).

21. N. Maccaferri, I. Zubritskaya, I. Razdolski, I.-A. Chioar, V. Belotelov, V. Kapaklis, P.M. Oppeneer, A. Dmitriev. Nanoscale magnetophotonics. J. Appl. Phys. 127, 080903 (2020).

22. R. Ruppin. Surface polaritons of a left-handed medium. Phys. Lett. A 277, 61 (2000).

23. R. Ruppin. Surface polaritons of a left-handed material slab. J. Phys.: Condens. Matter 13, 1811 (2001).

24. Y.V. Bludov, J.N. Gomes, G.A. Farias, J. Fernández-Rossier, M.I. Vasilevskiy, N.M.R. Peres. Hybrid plasmon-magnon polaritons in graphene-antiferromagnet heterostructures. 2D Mater. 6, 045003 (2019).

25. J. Sloan, N. Rivera, J.D. Joannopoulos, I. Kaminer, M. Soljačić. Controlling spins with surface magnon polaritons. Phys. Rev. B 100, 235453 (2019).

26. V.V. Temnov, G. Armelles, U. Woggon, D. Guzatov, A. Cebollada, A. Garcia-Martin, J.-M. Garcia-Martin, T. Thomay, A. Leitenstorfer, R. Bratschitsch. Active magnetoplasmonics in hybrid metal-ferromagnet structures. Nature Photon. 4, 107 (2010). 
27. D. Martín Becerra. Active Plasmonic Devices Based on Magnetoplasmonic Nanostructures (Springer, 2017).

28. G.T. Papadakis, D. Fleischman, A. Davoyan, P. Yeh, H.A. Atwater. Optical magnetism in planar metamaterial heterostructures. Nature Commun. 9, 296 (2018).

29. I. Zavislyak, H. Chumak. Magnon-plasmon polaritons in the layered structure metal-ferrite with a periodic stripelike structure of domains. Ukr. J. Phys. 64, 956 (2019).

30. S. Ramo, J. R. Whinnery, T. Van Duzer. Fields and Waves in Communication Electronics. (Wiley, 1994).

31. C. Kittel. Introduction to Solid State Physics (Wiley, 2004).

32. J. Lindhard. On the properties of a gas of charged particles. Mat. Fys. Medd. Dan. Vid. Selsk. 28, 1 (1954).

33. A.G. Gurevich, G.A. Melkov. Magnetization Oscillations and Waves (CRC Press, 1996).

34. M.A. Ordal, R.J. Bell, R.W. Alexander, Jr, L.L. Long, M.R. Querry. Optical properties of fourteen metals in the infrared and far infrared: $\mathrm{Al}, \mathrm{Co}, \mathrm{Cu}, \mathrm{Au}, \mathrm{Fe}, \mathrm{Pb}, \mathrm{Mo}, \mathrm{Ni}$, Pd, Pt, Ag, Ti, V, and W. Appl. Opt. 24, 4493 (1985).

Received 12.07.20. Translated from Ukrainian by O.I. Voitenko
В.Ю. Малишев, І.В. Зависляк

Г.А. Мелков, М.О. Попов, О.В. Прокопенко

МІКРОХВИЛЬОВІ

МАГНОН-ПЛАЗМОН-ПОЛЯРИТОНИ

В СТРУКТУРІ ФЕРОМАГНІТНИЙ

МЕТАЛ-ЕКРАНОВАНИЙ ДІЕЛЕКТРИК

Р е $з$ ю м е

Теоретично проаналізовано можливість існування поверхневих магнон-плазмон-поляритонів (ПМПП) - зв'язаних мікрохвильових коливань намагніченості, електронної густини та електромагнітного поля - в структурі реальний феромагнітний метал-діелектрик-ідеальний немагнітний метал. Розроблена теорія передбачає, що ефективне утворення ПМПП можливе лише за певних значень зовнішнього сталого магнітного поля і має супроводжуватися зсувом власної частоти резонансних плазмон-поляритонних систем. Теоретична оцінка величини цього зсуву для ПМПП в структурі резонатор поверхневої електромагнітної хвилі, виготовлений з пермалою,-вакуум-ідеальний метал сягає приблизно \pm 45 МГц для резонатора 3 власною частотою 10 ГГц, чого має бути достатньо для експериментального спостереження вказаного ефекту. 